Anna Anetta Janowska

Warsaw School of Economics

Institute for International Studies

\title{
L'avenir de la musique après la révolution numérique : opportunités et contraintes pour l'industrie du disque
}

Résumé : La révolution numérique est un phénomène assez récent, elle a cependant influencé très fort les industries créatives, parmi lesquelles l'industrie du disque. Assimilée souvent au piratage et donc considérée comme nocive aux productions musicales, elle a pourtant apporté des solutions positives aux auteurs, producteurs et distributeurs de la musique enregistrée. Dans le texte, à part le piratage, nous allons présenter quelques opportunités qui ont vu le jour sous la révolution numérique et qui se sont offertes aux acteurs de l'industrie du disque, devenant la source de profonds changements dans ce secteur du marché.

Mots-clés: révolution numérique, piratage, p2p, musique numérique, mp3, démocratisation, barrières à l'entrée, diversification, long tail

Abstract: The digital revolution is a relatively recent phenomenon, however, it has strong influence on the creative industries and the recorded music industry. It is often equated to piracy and for that reason considered harmful to musical productions. Yet it has brought positive solutions to authors, producers and distributors of recorded music. In the text, in addition to piracy, we will present some opportunities that have emerged with the digital revolution and that are available to actors of the music industry, becoming the source of profound changes in this market sector.

Keywords: digital revolution piracy, p2p, digital music, mp3, democratization, entry barriers, diversification, long tail. 
D'après les estimations de l'International Federation of Phonographic Industry représentant l'industrie du disque internationale, qui analyse le marché de la musique enregistrée et en publie régulièrement des rapports, en 2009, 95\% de la musique distribuée en format numérique était distribuée de façon illégale. Pour comparer, en 1999, donc au moment où la révolution numérique commençait seulement à être présente dans le monde des produits culturels, le niveau de distribution illégale de la musique se situait au niveau de $36 \%{ }^{1}$.

En fait, une des premières idées qui vient à l'esprit lorsque l'on mentionne les deux notions du titre de l'article : révolution numérique et industrie du disque, est le piratage. L'industrie du disque et surtout les majors ${ }^{2}$, détenant environ $70 \%$ du marché global de la musique enregistrée ${ }^{3}$, considèrent le piratage comme la première cause de la diminution des revenus de l'industrie suite à la chute des ventes des disques sur le marché global. L'IFPI, dans son rapport sur le piratage, constate qu'il est également responsable de l'érosion de la culture, qu'il déstabilise l'activité économique, prive l'état des revenus des taxes provenant de la vente de la musique légale et provoque des pertes au niveau des investissements ${ }^{4}$. II nuit à la créativité et à la diversité de l'offre musicale. Bref, il est considéré comme la plus grande contraine au développement actuel de l'industire du disque.

Le piratage dans l'industrie du disque n'est pourtant pas un phénomène récent. II est né avec la casette audio. Déjà à ce moment-là (dans les années 70 ), l'industrie du disque a accusé les pirates de la crise des ventes.

Lorsque le $C D$ a vu le jour, au début des années 80 , le piratage a augmenté. La musique - enregistrée en format numérique - a pu être copiée de façon parfaite, ce qui veut dire que la qualité sonore de la copie était identique à celle de l'original. Tout cela n'a pas empeché l'industrie du disque de connaître un très fort développement qui a duré depuis le début des années 80 jusqu'à la fin des années

\footnotetext{
${ }^{1}$ IFPI, Digital Music Report 2009, http://www.ifpi.org/content/library/Piracy2000.pdf, http://www.ifpi.org/content/library/DMR2009.pdf.

${ }^{2}$ On appelle les „majors” les quatre maisons de disques multinationales : Universal Music Group, Sony Music Entertainment, EMI, Warner Music.

${ }^{3}$ Laing (D.), World Music and the Global Music Industry: Flows, Corporations and Networks (pdf), ed. Tuulikki (P.), World Music: Roots and Routes. Studies across Disciplines in the Humanities and Social Sciences 6., Helsinki Collegium for Advanced Studies, Helsinki 2009, p. 15.

${ }^{4}$ IFPI, Music Piracy Report 2000, http://www.ifpi.org/content/library/Piracy2000.pdf.
} 
90 environ. C'est en 1988 que les ventes de la musique enregistrée ont été les plus grandes dans l'histoire, notamment aux Etats-Unis et au Royaume-Uni.

Le développement de l'internet et du format compressé de la musique, dont le plus populaire est le mp3, accompangés de l'invention des systèmes d'échange des fichiers numériques $\mathrm{p} 2 \mathrm{p}$ (peer-to-peer, pareil-à-pareil) ${ }^{5}$ ont provoqué un phénomène global des échanges incontrôlés, donc illégaux, de la musique entre les internautes. L'augmentation du niveau du piratage est donc un fait. II reste à savoir s'il est effectivement responsable de la chute des ventes des disques, comme le prétendent les maisons de disques. Les opinions à ce sujet restent partagées. Certains chercheurs, comme Peitz et Waelbroeck ${ }^{6}$, ont démontré que la chute des ventes des CD à cause du téléchargement des mp3 était de -20\% entre 1998 et 2002. II ont en même temps souligné que d'autres facteurs, à part le piratage, ont pu provoquer cette chute, par exemple le développement des autres formes de l'activité en ligne (créer du contenu en ligne, regarder des clip vidéo et films, jouer à des jeux en ligne, faire des achats, rechercher des informations). Liebowitz ${ }^{7}$ a confirmé la susdite analyse en concluant qu'il existait un lien étroit entre le piratage et la situation de crise dans laquelle se trouvait l'industrie du disque, il était cependant difficile de mesurer les effets de cette interdépendance.

L'étude menée par Oberholzer et $\operatorname{Strumpf}^{8}$ a donné des résultats totalement opposés. Selon ces chercheurs, l'échange des fichiers musicaux sur l'internet n'avait pas d'impact qui serait statistiquement significatif sur la chute des ventes des disques. Selon l'analyse, d'autres facteurs y ont joué un rôle important, parmi lesquels une situation macroéconomique qui serait défavorable pour les industries culturelles, la diminution du nombre de sorties des albums, la diminution de la variété

\footnotetext{
${ }^{5}$ p2p (angl. peer-to-peer, égal à égal) - ce sont des réseaux d'échange des fichiers sur Internet. Chaque utilisateur du réseau, s'il veut participer à ce systèle, installe sur son ordinateur un logiciel spécial via lequel il peut avoir accès au contenu se trouvant sur les disques durs des autres utilisateurs du même système. Avec les réseaux $\mathrm{p} 2 \mathrm{p}$, les interantes échangent avant tout des fichiers musicaux, mais également des films, des jeux, des logiciels.

${ }^{6}$ Peitz (M.), Waelbroeck (P.), The Effect of Internet Piracy on Music Sales: Cross-Section Evidence, Review of Economic Research on Copyright Issues (pdf), 2004, 1 (2), pp. 71 - 79.

${ }^{7}$ Liebowitz (S.), Will MP3 Downloads Annihilate the Record Industry? The Evidence so Far (pdf), Advances in the Study of Entrepreneurship, Innovation and Economic Growth, Gary Libecap ed. JAI Press, http://papers.ssrn.com/sol3/papers.cfm?abstract_id=414162, June 2003.

${ }^{8}$ Oberholzer (F.), Strumpf (K.), The Effect of File Sharing on Record Sales. An Empirical Analysis (pdf), Harvard Business School \& UNC Chapel Hill, http://www.unc.edu/ cigar/strumab.htm, March 2004.
} 
de l'offre musicale, liée à une forte consolidation de la radio, ainsi que les facteur qui ont déjà été rappelés par Peitz et Waelbroeck.

Dans la lumière des susdites analyses, il serait ainsi éronné d'assimiler la révolution numérique au piratage et encore moins de la considérer comme source des éléments uniquement nocifs au développement du marché de la musique enregistrée. Ella a en fait donné naissance à certains phénomènes - dans la production, la distribution et la promotion de la musique - qui ont changé les rapports entre différents acteurs de l'industrie de la musique enregistrée et qui peuvent être évalués comme positifs.

En premier lieu, il faut citer la démocratisation des outils de production de la musique. On estime qu'uniquement en 2008, 1 mld d'ordinateurs étaient en usage dans le monde et selon les prévisions de l'instutut d'analyses - Gartner - ce nombre atteindra 2 mld en $2014^{9}$. En même temps, 1 mld de personnes vivent aujourd'hui dans des sociétés suffisamment riches pour profiter des ICT, disposant de 2 à 6 mld d'heures de temps libre. Ces conditions-ci permettent donc à ces gens de créer du contenu, entre autres de la musique, à des frais très bas et à la qualité numérique. Cette musique peut donc être enregistrée, travaillée et copiée sur l'ordinateur, ensuite gravée sur $C D$, sans qu'une maison de disques nécessite d'être engagée dans tout ce processus pour assurer du matériel et du personnel spécialisés.

En deuxième lieu, il s'agit de la démocratisation des outils de distribution, étant à l'origine d'une baisse importante des barrières à l'entrée. Produire de la musique n'est en fait que la première étape de tout le cycle. L'artiste doit créer et produire, mais pour que son activité artistique ait du sens, il doit aussi être disitribué et connu par les auditeurs. Dans le modèle traditionnel, la distribution - surtout réalisée à l'échelle internationale, ou même globale - était une activité coûteuse et, en plus, contrôlée en presque 95\% par les majors ${ }^{10}$. Deux innovations récentes : le format mp3 et le développement de l'Internet ont contribué à cette démocratisation de la distribution. Grâce à la dématérialisation du support, les oeuvres musicales peuvent être sauvegardées sur le disque dur en format compressé - mp3 - et

\footnotetext{
${ }_{9}^{9}$ Eskelsen (G.), Marcus (A.), Kenneth Ferree (W.), The Digital Economy Fact Book (pdf), Tenth Edition, The Progress \& Freedom Foundation, 2009, http://www.pff.org/issues-pubs/books/factbook 10th Ed.pdf

${ }^{10}$ Alexander (P.J.), Peer-to-Peer File Sharing: The Case of the Music Recording Industry, Review of Industrial Organization, 2002, 20, p. 153.
} 
distribuées à travers le réseau global presque sans coûts. L'Internet offre donc aux artistes non seulement un canal de distrubution bon marché, mais aussi - avec la desintermédiation qu'il assure - il leur permet de toucher directement le public.

La baisse des barrières à l'entrée au niveau de la distribution a permis aux nouveaux acteurs d'apparaître sur le marché de la musique enregistrée : c'est en fait une certaine réintermédiation qui a pu avoir lieu. Tout d'abord, des magasins de vente en ligne se sont installés, offrant de la musique sur CD. Celui qui a connu le plus grand succès et une expansion globale est Amazon.

En 2003, la société Apple, produisant jusqu'alors des ordinateurs, a créé un plateau de distribution de la musique en ligne, en format numérique - iTunes. Actuellement, c'est le plus grand vendeur en détail de la musique en format mp3 et similaires, détenant 25\% du marché aux USA. Ses revenus provenant de la vente de la musique étaient en 2009 de 1,8 mld USD et le nombre de titres téléchargés a dépassé $10 \mathrm{mld}^{11}$. Aujourd'hui, le nombre de plateaux de vente en ligne est de plus de 400. Les revenus globaux des ventes en 2009 étaient de 4,2 mld USD (27\% de la valeur totale des ventes de la musique) ${ }^{12}$. Ce chiffre augmente chaque année.

L'apparition de ces nouveaux plateaux de distribution a provoqué une déconcentration de la distribution de la musique, ce qui a augmenté le niveau de concurrence entre eux et a contribué à la baisse des barrières à l'entrée pour de petits producteurs. II est vrai que la plupart des plateaux proposent avant tout la musique provenant des majors, mais il y en a aussi qui offrent des albums produits par des labels indépendants, comme eMusic.com, le plus grand service de ce genre, dont le catalogue compte 6 mln chansons, provenant de plus de 60 mille labels ${ }^{13}$.

Grâce à la démocratisation de la distribution de la musique, l'offre de celle-ci, accessible en ligne, a augmenté de façon considérable. Anderson a appelé ce phénomène « long tail » (longue traîne). Selon la théorie portant le même nom qu'il a développé en $2004^{14}$, l'Internet a aidé à développer une mosaïque de mini-marchés et de micro-stars, le marché de masse se transformant en un nouveau marché de

\footnotetext{
${ }^{11}$ Apple iTunes Store Revenue, http://gorumors.com/crunchies/apple-itunes-revenue/, accès août 2010.

${ }_{12}^{12}$ IFPI, Digital Music Report 2010, http://www.ifpi.org/content/library/DMR2010.pdf.

${ }^{13}$ http://www.emusic.com/about/index.html.

${ }^{14}$ The Long Tail, http://www.wired.com/wired/archive/12.10/tail.html?pg=5, accès août 2010.
} 
niches qui - pour la première fois - peut entrer en compétition avec le marché des tubes $^{15}$.

En troisième lieu, les changements que la révolution numérique a entraînés, ont également eu lieu au niveau de la promotion et surtout d'une de ses fonctions l'information. Les barrières à l'entrée aux canaux de promotion dans le modèle traditionnel étaient aussi hautes que celles pour la distribution de la musique. Les artistes individuels et des labels indépendants n'ont jamais pu assurer une promotion importante de leurs productions, l'échelle de celle-ci restant locale ou bien de niche. En ce qui concerne les albums produits par des majors, ce ne sont pas non plus tous qui ont pu trouver leur place dans les médias. Les coûts élevés de promotion étaient la cause principale de cet état des choses, l'autre étant le temps restreint disponible à l'antenne pour diffuser les nouveautés.

L'Internet a ainsi offert des opportunités à toutes ces productions d'être présentées au large public à travers des sites Internet des artistes, des forums de discussion, des services sociaux etc. Un des plus grands services, créé pour promouvoir la musique indépendante, était MySpace. Selon les estimations, en 2009, on pouvait y trouver $20 \mathrm{mln}$ de chansons et $100 \mathrm{mln}$ d'utilisateurs actifs environ ${ }^{16}$.

La démocratisation des moyens et canaux de promotion a permis au nombre beaucoup plus élevé d'artistes et petites maisons de disques - par rapport aux conditions du modèle traditionnel - de se faire connaître par un large public, ceci au côut presque nul. Le résultat de cette liberté d'information était une abondance infinie d'oeuvres, parmi lesquelles il est devenu de plus en plus difficile de trouver celle qui pouvait correspondre le mieux aux préférences.

L'Internet a apporté également des solutions à ce problème de bruit informationnel et de coût de plus en plus élevé de la recherche d'informations par les consommateurs. La première sont des moteurs de recherche, p.ex. Google. Parmi d'autres, on peut citer des utilités proposées par les magasins en ligne où l'acheteur

\footnotetext{
${ }^{15}$ Anderson (C.), Długi ogon. Ekonomia przyszłości - każdy konsument ma głos, Poznań, Media Rodzina, 2008, p. 37. Trad. d'anglais B. Ludwiczak (The Long Tail. Why the Future of Business is Selling Less of More), p. 257. ${ }^{16}$ MySpace's Dwindling Traffic Looks Even Worse From the Inside, http://www.dailyfinance.com/story/company-news/myspaces-dwindling-traffic-looks-even-worse-from-theinside/19380431/, accès août 2010.
} 
est invité, parfois contre un petit rabais sur l'achat suivant, à laisser une évaluation de l'album ou la chanson achetés. Les mécanismes internes de ces plateaux collectent également des informations sur leurs clients, ce qui permet de proposer aux autres, choissant un produit donné, un autre produit, acheté par un consommateur ayant des préférences similaires.

A part les solutions techniques, le réseau global à aidé à la formation de la génération du web 2.0., donc des groupes de consommateurs prêts à offrir aux autres utilisateurs, gratuitement, des informations sur la musique qu'ils aimaient, des opinions, comptes rendus, playlistes, classements etc. Ce système de «bouche à oreille », lié à «l'effet de mode» (bandwagon effect) ${ }^{17}$, est un des éléments principaux qui influencent les décisions d'achat de la musique, à cause de son caractère de « bien d'expérience ${ }^{18}$.

Benkler souligne également ${ }^{19}$ le fait que les réseaux $\mathrm{p} 2 \mathrm{p}$, considérés par l'industrie du disque comme outils de piratage, peuvent être regardés sous un autre angle. Ils sont pour lui des réseaux de distribution de la musique numérique en ligne, ayant un caractère global et - avant tout - gratuit. L'industrie de la musique enregistrée, si elle avait l'intention d'installer un tel réseau par elle-même, devrait investir des sommes importantes pour acheter des serveurs, disques durs et accès Internet à grande capacité. Dans le cas des réseaux en question, les internautes couvrent ces frais eux-mêmes, en contribuant à la propagation - sans limites de temps et d'espace - de la musique et des informations sur la musique. Ils agissent conformément à l' « effet réseau ${ }^{20}$, dans lequel plus d'utilisateurs participent au réseau, plus grande est l'utilité de celui-ci.

\footnotetext{
${ }^{17}$ Leibenstein (H.), Bandwagon, Snob, and Veblen Effects in the Theory of Consumers' Demand (pdf), The Quarterly Journal of Economics, Vol. 64, N² 2 , mai 1950, p. 183-207, http://links.jstor.org/sici?sici=00335533\%28195005\%2964\%3A2\%3C183\%3ABSAVEI\%3E2.0.CO\%3B2-Q.

${ }^{18}$ Nelson (P.), Information and Consumer Behavior, Journal of Political Economy, 78, 1970, pp. 311-329; Solomon (M.R.), Bamossy (G.), Askegaard (S.), Hogg (M.K.), Consumer Behaviour. A European Perspective, Essex, Pearson Education, 2010. Le bien d'expérience est un bien qui - avant d'être consommé - doit être écouté, regardé ou senti.

${ }^{19}$ Benkler, Y., Bogactwo sieci. Jak produkca społeczna zmienia rynki i wolność, Warszawa, WAiP, 2008, p. 116. Trad. d'anglais R. Próchaniak (The Wealth of Networks. How Social Production transforms Markets and Freedom).

${ }^{20}$ Hammond (T.), Hannay (T.), Lund (B.), Scott (J.), Social Bookmarking Tools. A general review (pdf), D-Lib Magazine, avril 2005, http://www.dlib.org/dlib/april05/hammond/04hammond.html.
} 
Il est difficile de donner raison à Benkler, surtout en tenant compte de la forme du droit d'auteur en vigueur. Les limites de cet article ne nous permettent pas de développer la question de la justesse des solutions juridiques actuelles par rapport aux biens numériques, il est pourtant important d'attirer l'attention sur le fait que la révolution numérique, à par les effets strictement économiques, telles que la baisse des coûts et par conséquent des barrières à l'entrée, contibuera peut-être aux changements au niveau du droit de la propriété intellectuelle. Ceci devra entraîner par la suite le développement des nouveaux modèles d'affaires, ou même un nouveau modèle économique concernant la production, la distribution et la promotion de la musique enregistrée.

\section{Bibliographie:}

Alexander (P.J.), Peer-to-Peer File Sharing: The Case of the Music Recording Industry (pdf), Review of Industrial Organization, 2002, 20.

Anderson (C.), Długi ogon. Ekonomia przyszłości - każdy konsument ma głos, Poznań, Media Rodzina, 2008, p. 37. Trad. d'anglais B. Ludwiczak (The Long Tail. Why the Future of Business is Selling Less of More).

Benkler, Y., Bogactwo sieci. Jak produkcja społeczna zmienia rynki i wolność, Warszawa, WAiP, 2008. Trad. d'anglais R. Próchaniak (The Wealth of Networks. How Social Production transforms Markets and Freedom).

Eskelsen (G.), Marcus (A.), Kenneth Ferree (W.), The Digital Economy Fact Book (pdf), Tenth Edition, The Progress \& Freedom Foundation, 2009, http://www.pff.org/issues-pubs/books/factbook 10th Ed.pdf

Hammond (T.), Hannay (T.), Lund (B.), Scott (J.), Social Bookmarking Tools. A general review (pdf), D-Lib Magazine, avril 2005, http://www.dlib.org/dlib/april05/hammond/04hammond.html.

Laing (D.), World Music and the Global Music Industry: Flows, Corporations and Networks (pdf), ed. Tuulikki (P.), World Music: Roots and Routes. Studies across Disciplines in the Humanities and Social Sciences 6., Helsinki Collegium for Advanced Studies, Helsinki 2009.

Leibenstein (H.), Bandwagon, Snob, and Veblen Effects in the Theory of Consumers' Demand (pdf), The Quarterly Journal of Economics, Vol. 64, N² 2 , mai 1950, http://links.jstor.org/sici?sici=00335533\%28195005\%2964\%3A2\%3C183\%3ABSAVEI\%3E2.0.CO\%3B2-Q. 
Liebowitz (S.), Will MP3 Downloads Annihilate the Record Industry? The Evidence so Far (pdf), Advances in the Study of Entrepreneurship, Innovation and Economic Growth, Gary Libecap ed. JAI Press,

http://papers.ssrn.com/sol3/papers.cfm?abstract_id=414162, June 2003.

Nelson (P.), Information and Consumer Behavior, Journal of Political Economy, 78, 1970.

Oberholzer (F.), Strumpf (K.), The Effect of File Sharing on Record Sales. An Empirical Analysis (pdf), Harvard Business School \& UNC Chapel Hill, http://www.unc.edu/ cigar/strumab.htm, March 2004.

Peitz (M.), Waelbroeck (P.), The Effect of Internet Piracy on Music Sales: CrossSection Evidence, Review of Economic Research on Copyright Issues (pdf), 2004, 1 (2).

Solomon (M.R.), Bamossy (G.), Askegaard (S.), Hogg (M.K.), Consumer Behaviour. A European Perspective, Essex, Pearson Education, 2010.

\section{Sources électroniques :}

Apple iTunes Store Revenue, http://gorumors.com/crunchies/apple-itunes-revenue/.

http://www.emusic.com/about/index.html.

IFPI, Digital Music Report 2009, http://www.ifpi.org/content/library/Piracy2000.pdf, http://www.ifpi.org/content/library/DMR2009.pdf.

IFPI, Digital Music Report 2010, http://www.ifpi.org/content/library/DMR2010.pdf.

IFPI, Music Piracy Report 2000, http://www.ifpi.org/content/library/Piracy2000.pdf.

MySpace's Dwindling Traffic Looks Even Worse From the Inside, http://www.dailyfinance.com/story/company-news/myspaces-dwindling-traffic-lookseven-worse-from-the-inside/19380431/.

The Long Tail, http://www.wired.com/wired/archive/12.10/tail.html?pg=5. 